\title{
Uric acid and contrast-induced nephropathy: an updated review and meta-regression analysis
}

\author{
Francesco Pelliccia ${ }^{1}$, Vincenzo Pasceri ${ }^{2}$, Giuseppe Patti ${ }^{3}$, Giuseppe Marazzi ${ }^{4}$, Giuseppe De Luca ${ }^{5}$, \\ Gaetano Tanzilli ${ }^{1}$, Nicola Viceconte ${ }^{1}$, Giulio Speciale ${ }^{2}$, Enrico Mangieri ${ }^{1}$, Carlo Gaudio ${ }^{1}$ \\ ${ }^{1}$ Department of Cardiovascular Sciences, Sapienza University, Rome, Italy \\ ${ }^{2}$ Cardiovascular Intervention, San Filippo Neri Hospital, Rome, Italy \\ ${ }^{3}$ University of L'Aquila, L'Aquila, Italy \\ ${ }^{4}$ IRCCS San Raffaele Pisana, Rome, Italy \\ ${ }^{5}$ Division of Cardiology, Azienda Ospedaliera-Universitaria “Maggiore della Carità”, Eastern Piedmont University, Novara, Italy
}

Adv Interv Cardiol 2018; 14, 4 (54): 399-412

DOI: https://doi.org/10.5114/aic.2018.79870

\begin{abstract}
A bstract
Introduction: Previous studies have suggested a relationship between serum uric acid and contrast-induced nephropathy (CIN). Aim: We performed an updated review and a meta-regression analysis to assess whether serum uric acid is associated with CIN or there exists any relationship between serum uric acid and other risk factors for CIN.

Material and methods: We searched PubMed, Embase and Cochrane databases and reviewed cited references up to July 31 , 2018 to identify relevant studies.

Results: A total of 6,705 patients from 10 clinical studies were included. CIN occurred in 774 of the 6,705 (12\%) patients. Baseline uric acid levels were significantly higher in those who developed CIN (6.51 vs. $5.67 \mathrm{mg} / \mathrm{dl} ; 95 \% \mathrm{Cl}: 0.55-1.22, p=0.00001)$. Comparison of clinical features showed that patients with CIN were significantly older ( 69 vs. 63 years; $p<0.00001$ ) and more often had diabetes ( $42 \%$ vs. $32 \% ; p=0.002)$ and hypertension ( $67 \%$ vs. $59 \% ; p=0.03$ ). Also, patients who developed CIN had lower hemoglobin ( 12.5 vs. $13.6 \mathrm{mg} / \mathrm{dl} ; p<0.00001)$ and higher levels of baseline creatinine (1.27 vs. $1.01 \mathrm{mg} / \mathrm{dl} ; p<0.0001)$, but had similar levels of glycemia, total cholesterol, low-density lipoprotein cholesterol, high-density lipoprotein cholesterol, and triglyceride. Also, they showed a lower ejection fraction ( $45 \%$ vs. $50 \%$; $p<0.00001)$. Meta-regression analysis revealed that uric acid related only to age $(r=0.13, p=0.03)$.

Conclusions: Our investigation indicates that uric acid is significantly associated with CIN. Uric acid correlated significantly with age only, and not with other major predictors of CIN. Further studies are therefore needed to verify the potential of uric acid to improve CIN risk stratification.
\end{abstract}

Key words: angiography, contrast-induced nephropathy, percutaneous coronary intervention, uric acid.

S u m m a ry

Uric acid relates to the occurrence of contrast-induced nephropathy (CIN), but it remains unclear whether it is merely related to traditional risk factors for CIN or plays an independent predictive role. We performed a meta-analysis and a metaregression analysis of 10 clinical studies. Baseline uric acid levels were significantly higher in those who developed CIN. Statistical analysis revealed that uric acid correlated significantly with age only, but not with other major predictors of CIN, such as diabetes, hypertension, hemoglobin, creatinine, glycemia, cholesterol, and triglycerides. Uric acid seems to have the potential to improve CIN risk stratification.

\section{Introduction}

Contrast-induced nephropathy (CIN) is one of the most common complications of procedures that require the use of contrast media [1, 2]. Contrast-induced ne- phropathy occurs in up to $25 \%$ of patients undergoing diagnostic and/or therapeutic coronary angiography, depending on the presence of known risk factors, such as chronic kidney disease, diabetes mellitus, high dose of

\section{Corresponding author:}

Francesco Pelliccia MD, PhD, Department of Cardiovascular Sciences, Sapienza University, Viale Del Policlinico 155,00161 Rome, Italy, phone: +39348 3392006, fax: +3906330 62516, e-mail: f.pelliccia@mclink.it

Received: 16.09 .2018 , accepted: 8.10.2018. 
contrast medium, congestive heart failure, and anemia $[3,4]$. In recent years, several investigations and some systematic reviews and meta-analyses have shown that hyperuricemia relates to a greater frequency of CIN [5-7]. However, no previous investigation has assessed whether serum uric acid plays an independent role in CIN stratification or is merely related to presenting features and procedural characteristics of patients undergoing elective coronary angiography.

\section{Aim}

The aim of the current study was to perform an updated review and a meta-regression analysis in order to assess whether serum uric acid is associated with CIN or there exists any relationship between serum uric acid and other risk factors for CIN.

\section{Material and methods}

\section{Study design}

This meta-analysis was conducted following current guidelines, including the Cochrane Collaboration and Meta-analysis Of Observational Studies in Epidemiology (MOOSE), and the Preferred Reporting Items for Systematic reviews and Meta-Analyses (PRISMA) amendment to the Quality of Reporting of Meta-analyses (QUOROM) statement [8-10]. All activities were carried out independently by two reviewers (FP and VP). Divergences were solved after consensus.

\section{Data sources}

We searched PubMed, Embase, and Cochrane databases as well as the National Institutes of Health Clinical Trials Registry (www.clinicaltrials.gov) up to July 31, 2018 to identify relevant studies. In addition, we searched the presentations at major cardiovascular scientific sessions including meetings of the American College of Cardiology, American Heart Association and European Society of Cardiology. Additional studies were searched in Google Scholar and Scopus. Editorials and reviews from major medical journals published within the last 5 years were also considered for further information on studies of interest. No language restriction was enforced in order to minimize the risk of publication bias. The following combinations of medical subject heading terms and text words were used: "contrast induced acute kidney injury", "contrast induced acute renal failure", "contrast nephropathy", "contrast induced nephropathy", "contrast induced nephrotoxicity", "contrast associated nephropathy", "contrast associated nephrotoxicity", and "radiocontrast induced nephropathy".

\section{Study selection}

Retrieved citations were first screened independently by two unblinded investigators (FP and GP) at the title and/or abstract level, with divergences resolved after consensus. Studies were screened in order to identify potentially suitable articles that should be assessed for eligibility as full texts. Notably, a detailed review of study authors, dates, and locations was used to exclude redundancy. Studies with overlapping data were identified, and in cases of apparent serial reporting of a particular patient cohort, only the publication with the largest number of patients was included in the meta-analysis. Only studies published in original articles in peer-reviewed scientific journals were taken into consideration. Studies were then selected according to the following explicit inclusion criteria (all had to be met for inclusion): (i) case series of patients undergoing coronary angiography and/or $\mathrm{PCl}$; (ii) comparison of clinical, laboratory and procedural features between patients with or without post-procedural evidence of CIN; (iii) baseline assessment of uric acid levels.

\section{Data extraction and quality}

Analysis was performed at the study level, as databases of the individual patients were not obtained. Data from each study were obtained by two independent reviewers (FP and GP) and entered into a structured spreadsheet. Disagreements were resolved by consensus. Dichotomous variables were extracted in absolute numbers and were recalculated when percentages were reported. Continuous variables were extracted and weighted means for the total study population were calculated. The primary endpoint was baseline uric acid levels in patients with or without CIN. Other endpoints were the differences in clinical, laboratory and procedural features between patients with and without CIN, with special emphasis on renal function. Study validity was tested according to recommendations distinguishing several sources of bias. In order to assess the quality of reporting of the studies, the standard Strengthening the Reporting of Observational Studies in Epidemiology checklist (STROBE) was used [11]. This checklist includes items assessing the study methodology, study type, study population, sample size, sample collection methods, statistical tests, and presentations.

\section{Data analysis and synthesis}

All analyses were performed using the Review Manager 5.2.10 software and Comprehensive Meta-analysis Version 3.1. Since heterogeneity of results was expected, the inverse variance method for random effects was used to estimate pooled risk ratios or relative risk for dichotomous variables or mean difference for continuous variables and 95\% confidence intervals $(\mathrm{Cl})$ [12]. We tested the heterogeneity of the included studies with Q statistics and the extent of inconsistency between results with $R^{2}$ statistics. Significant heterogeneity was considered present in the case of $l^{2}>50 \%$ and/or $p<0.10$ [13]. 
However, we did not exclude outliers based on heterogeneity since heterogeneity is expected in meta-analyses of observational studies [14]. Accordingly, we performed a leave-one-out sensitivity analysis for each parameter in order to evaluate the robustness of the results and the impact of each single study on the summary estimate of effect. Briefly, we recalculated pooled estimates multiple times, using a random-effects model, each time with the removal of a single study from the original group. We also performed a sensitivity analysis stratified by sample size to exclude the possibility of overestimating the role of uric acid due to the inclusion of small studies (defined as those with a sample size $<500$ patients). Presence of publication bias was estimated by the Rucker test (with arcsine transformation), which is best suited for binary outcomes and funnel plot analysis [15, 16]. Meta-regression analysis was conducted to assess whether there was a direct relationship between levels of uric acid and other variables associated with CIN [17]. Specifically, we performed a weighted random-effects meta-regression regressing uric acid and CIN against the difference of each variable. Results are reported as beta coefficients and two-sided $p$-values. Statistical significance was set at $p<0.05$ (two-tailed).

\section{Results}

\section{Search results}

From 2,046 initial citations that were retrieved from multiple databases, a total of 78 articles were analyzed as full reports according to predefined selection criteria. After excluding 68 studies because serum uric acid levels in patients with or without CIN were not reported, 10 investigations were finally deemed as eligible for inclusion in our systematic review (Figure 1) [18-27]. A total of 6,705 patients were included in the 10 studies. Selected studies were published between 2010 and 2015, and included series of patients from North America, Europe, and Asia (Table I). All included patients underwent either coronary angiography or percutaneous coronary intervention (PCI). Quality assessment by STROBE checklist revealed moderate to high quality in all studies (Table II).

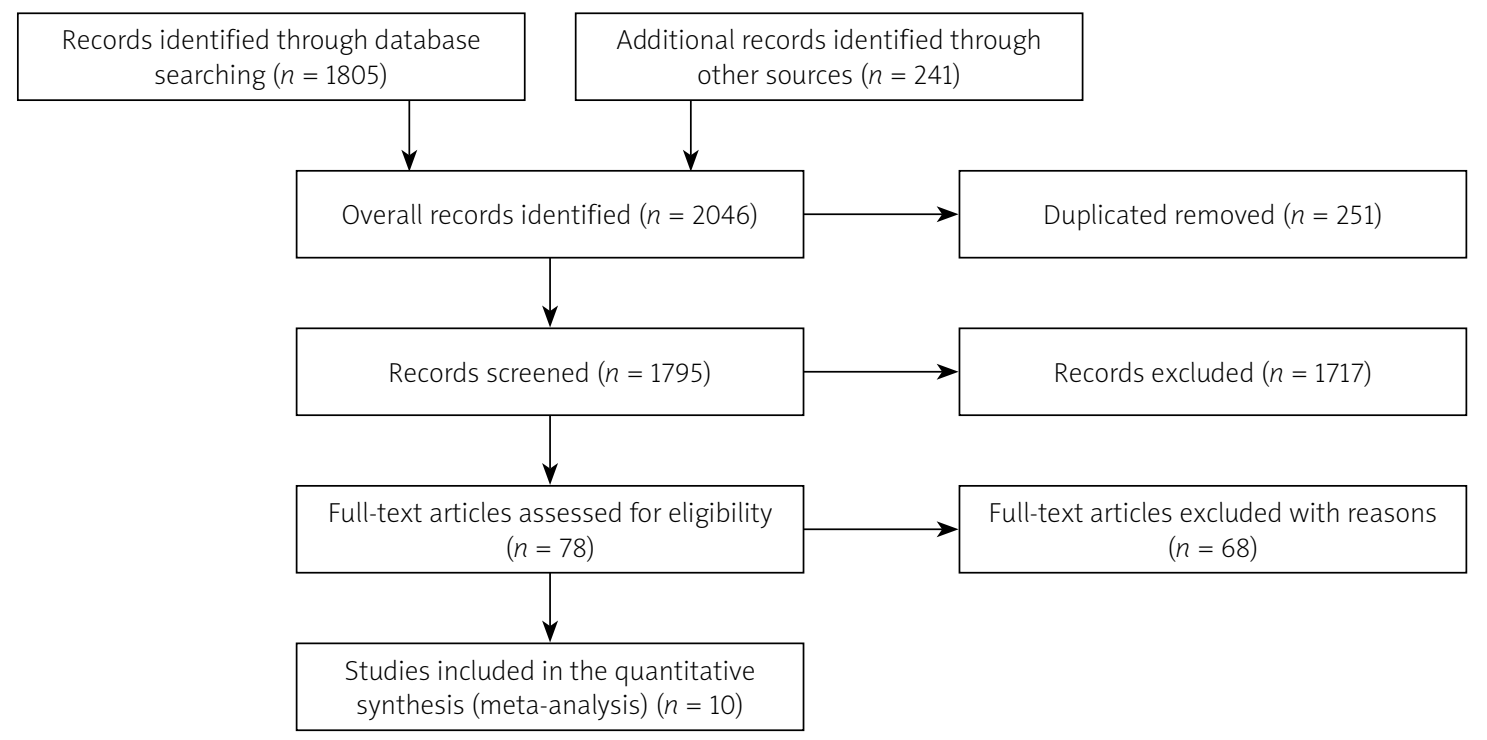

Figure 1. PRISMA diagram of study selection. The flow diagram demonstrates the study selection process in the systematic review and meta-analysis

Table I. List of included studies

\begin{tabular}{|c|c|c|c|c|c|c|c|}
\hline Author & Country & Overall $(n)$ & No CIN $(n)$ & CIN $(n)$ & Coro & $\mathrm{PCl}$ & Definition of CIN \\
\hline Parkfetrat & Iran & 290 & 245 & 45 & Yes & Yes & $\mathrm{SCr}>50 \%$ or $>0.5 \mathrm{mg} / \mathrm{dl}$ \\
\hline Okino & China & 139 & 130 & 9 & Yes & Yes & $\mathrm{SCr}>25 \%$ or $>0.5 \mathrm{mg} / \mathrm{dl}$ \\
\hline $\mathrm{Ma}$ & China & 69 & 43 & 26 & Yes & Yes & $\mathrm{SCr}>25 \%$ or $>0.5 \mathrm{mg} / \mathrm{dl}$ \\
\hline Park & Korea & 1247 & 1,196 & 51 & Yes & Yes & $\mathrm{SCr}>50 \%$ or $>0.5 \mathrm{mg} / \mathrm{dl}$ \\
\hline Kurtul & Turkey & 436 & 373 & 63 & Yes & Yes & $\mathrm{SCr}>25 \%$ or $>0.5 \mathrm{mg} / \mathrm{dl}$ \\
\hline Elbasan & Turkey & 835 & 755 & 80 & Yes & Yes & $\mathrm{SCr}>25 \%$ \\
\hline Saritemur & Turkey & 744 & 651 & 93 & Yes & Yes & $\mathrm{SCr}>25 \%$ or $>0.5 \mathrm{mg} / \mathrm{dl}$ \\
\hline $\mathrm{Ji}$ & China & 805 & 497 & 68 & Yes & Yes & $\mathrm{SCr}>25 \%$ or $>0.5 \mathrm{mg} / \mathrm{dl}$ \\
\hline Barbieri & Italy & 1950 & 1,699 & 251 & Yes & Yes & $\mathrm{SCr}>25 \%$ or $>0.5 \mathrm{mg} / \mathrm{dl}$ \\
\hline Karabulut & Poland & 430 & 342 & 88 & Yes & Yes & $\mathrm{SCr}>25 \%$ or $>0.5 \mathrm{mg} / \mathrm{dl}$ \\
\hline
\end{tabular}




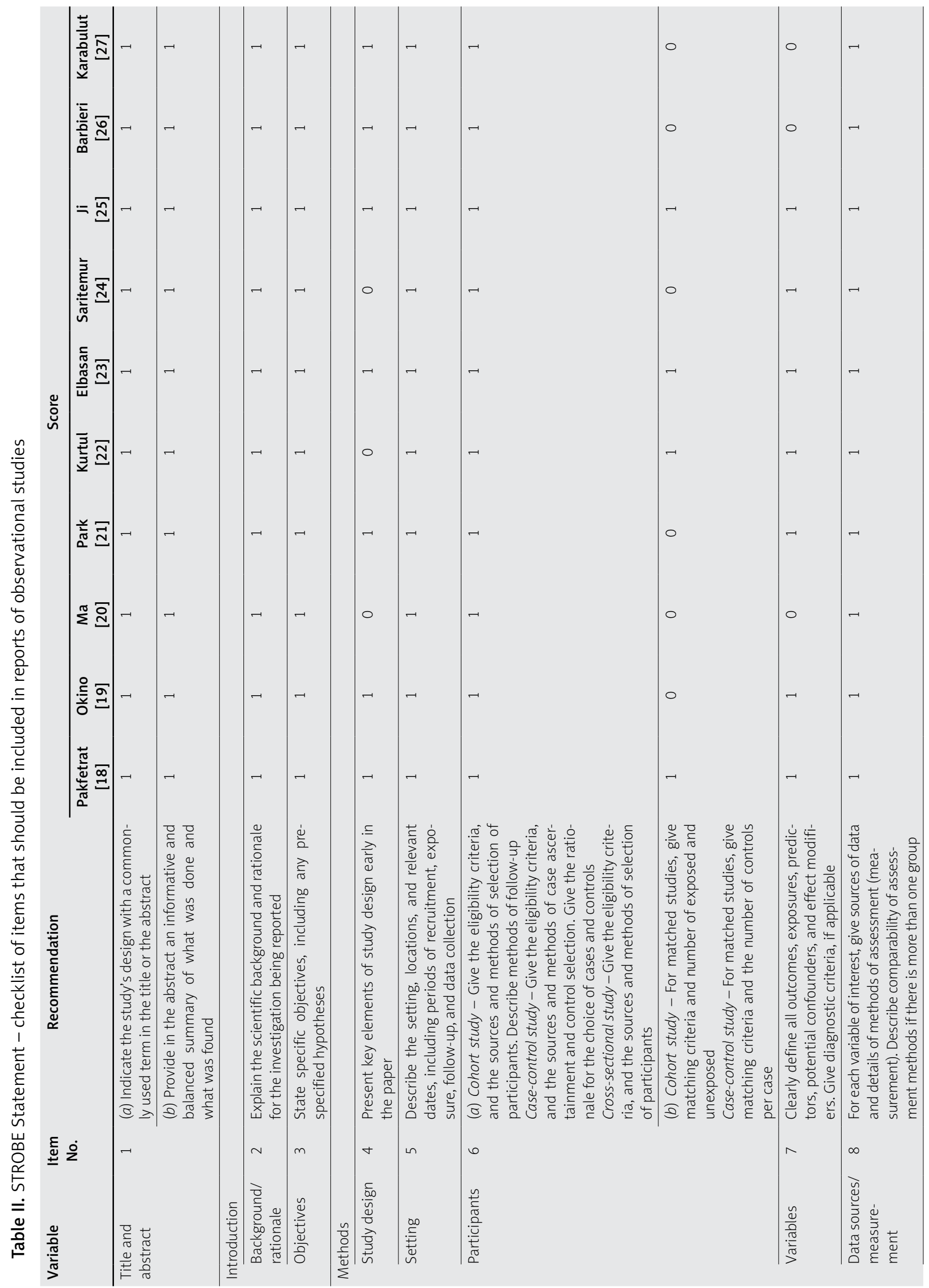




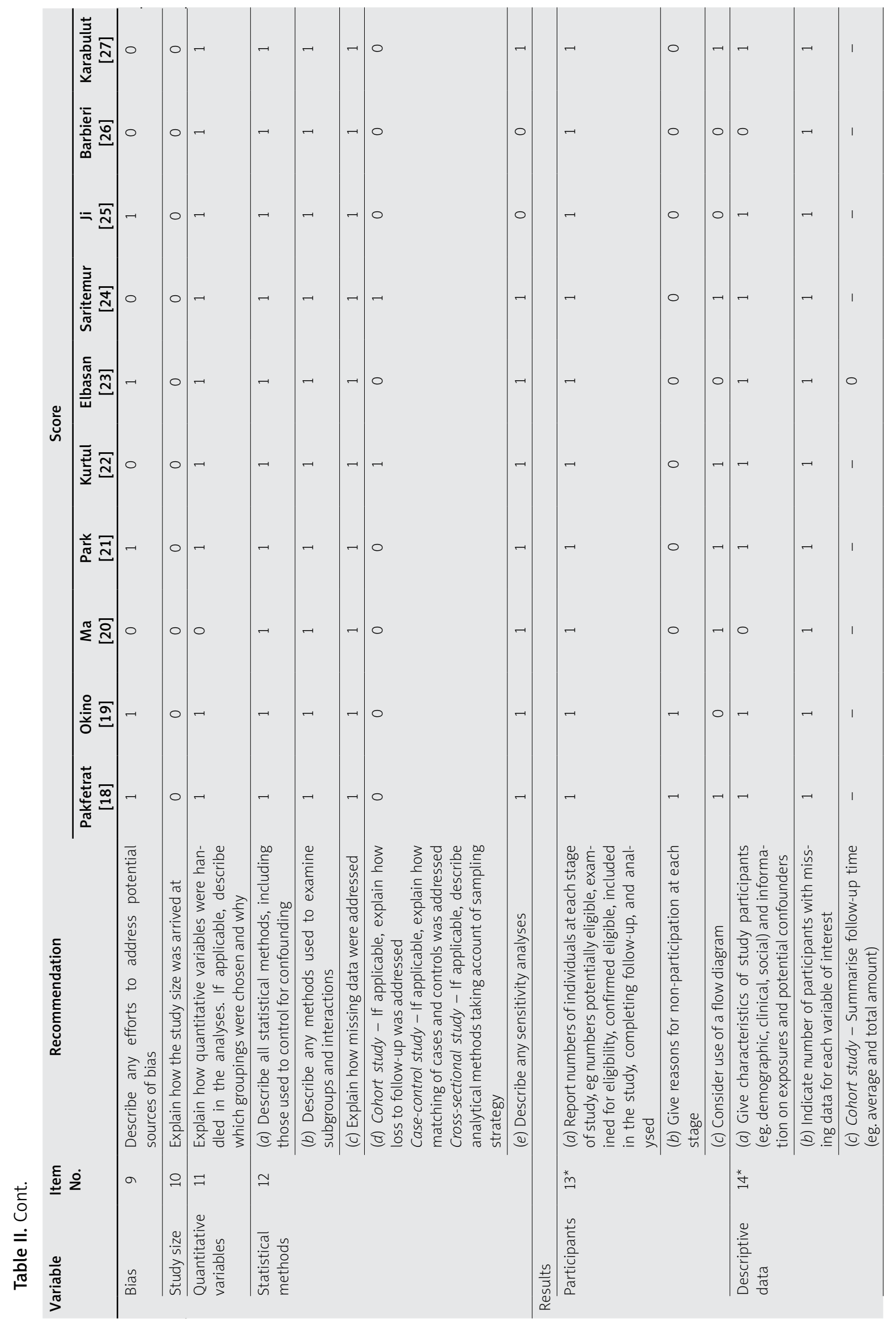




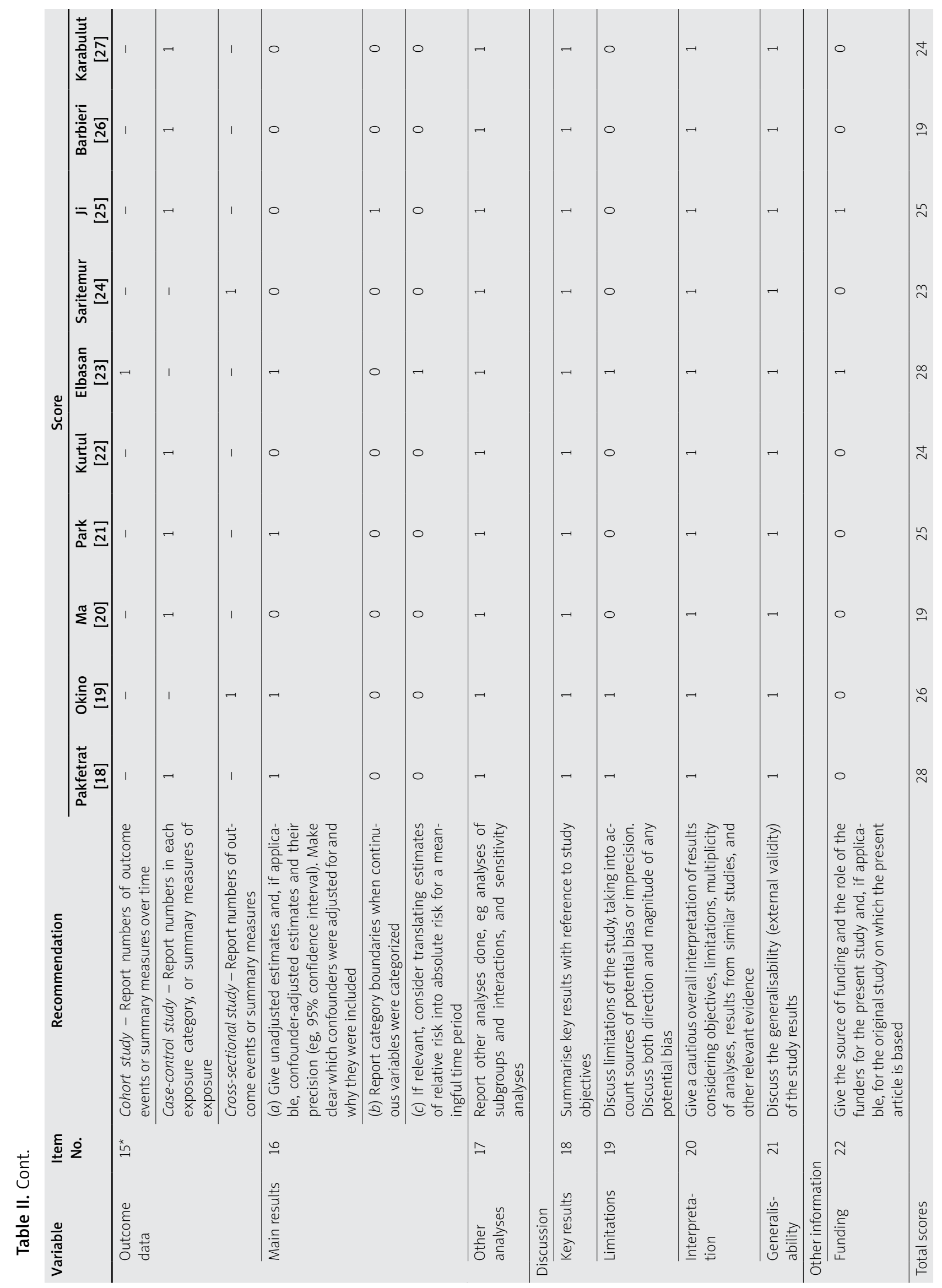




\section{Definition of CIN}

CIN was defined variously in the studies. Most studies described CIN as an increase in serum creatinine levels of more than $25 \%$ or $0.5 \mathrm{mg} / \mathrm{dl}$ above the baseline $48-72 \mathrm{~h}$ following intravascular contrast medium administration $[19,20,22,24-27]$. CIN was defined according to the RIFLE criteria by Pakfetrat et al. [18] as an increase in serum creatinine of $\geq 0.5 \mathrm{mg} / \mathrm{dl}$ or $\geq 50 \%$ over baseline within 7 days by Park et al. [21], and as an increase $\geq 25 \%$ in creatinine by Elbasan et al. [23].

\section{Primary end-point}

CIN occurred in 774 of the 6,705 (11.5\%) patients. Frequency of CIN ranged between $4.1 \%$ and $37.7 \%$. Baseline uric acid levels were significantly higher in those who developed CIN 6.51 (95\% Cl: 6.23-6.78) vs. 5.67 (95\% Cl: 5.25-6.09) mg/dl; mean difference: $0.88,95 \% \mathrm{Cl} 0.55-1.22$, $p<0.00001 ; l^{2}=83 \%, p<0.00001$ ) (Table III; Figure 2).

\section{Factors associated with CIN}

Comparison of clinical features at referral showed that patients who experienced CIN were significantly older (69 (95\% Cl: 64-72) vs. 63 (95\% Cl: 59-67) years; mean difference: $5.25,95 \% \mathrm{Cl}: 3.19-7.32, p<0.00001$; $\left.p^{2}=81 \%, p<0.00001\right)$, more often had diabetes $(42 \%$ vs. $32 \%$; risk ratio: $0.70,95 \% \mathrm{Cl}: 0.56-0.88, p=0.002$; $\left.P^{2}=81 \%, p<0.00001\right)$ and hypertension (67\% vs. $59 \%$; risk ratio: $0.84,95 \% \mathrm{Cl}: 0.72-0.98, p=0.03 ; l^{2}=81 \%$, $p<0.00001$ ), with no difference in prevalence of smoking and hyperlipidemia (Table III; Figure 3 ). With respect to laboratory findings at baseline, patients who developed $\mathrm{CIN}$ had lower hemoglobin $(12.5$ (95\% Cl: $12.1-12.9)$ vs. $13.6(95 \% \mathrm{Cl}: 13.2-13.9) \mathrm{mg} / \mathrm{dl}$; mean difference: 1.02 95\% Cl: 0.61-1.42, $\left.p<0.00001 ; p^{2}=71 \%, p=0.0002\right)$ and higher levels of baseline creatinine $(1.27$ (95\% Cl: 1.07-1.43) vs. 1.01 (95\% Cl: 0.86-1.16) mg/dl; mean difference: $0.22,95 \% \mathrm{Cl}: 0.11-0.32, p<0.0001 ; l^{2}=94 \%$, $p=0.00001$ ), but had similar levels of glucose, total cholesterol, low-density lipoprotein cholesterol, high-density lipoprotein cholesterol, and triglyceride (Table IV; Figure 3). Also, they showed a lower ejection fraction (45\% (95\% Cl: $42-47)$ vs. $50 \%$ (95\% Cl: 46-53); mean difference: $4.89,95 \% \mathrm{Cl}: 2.77-7.01, p<0.00001 ; l^{2}=71 \%$, $p=0.002$ ) (Table V; Figure 4).

\section{Meta-regression analysis}

Meta-regression (Table $\mathrm{VI}$ ) revealed that uric acid, apart from a relationship with age $(r=-0.75, p=0.02)$, was not associated with major risk factors for $\mathrm{CIN}$, i.e. diabetes, hypertension, ejection fraction, hemoglobin and baseline creatinine (Figures 5 and 6).

\section{Sensitivity analysis and publication bias}

The leave-one-out analysis showed that the pooled results were not influenced by any single trial. In addi-

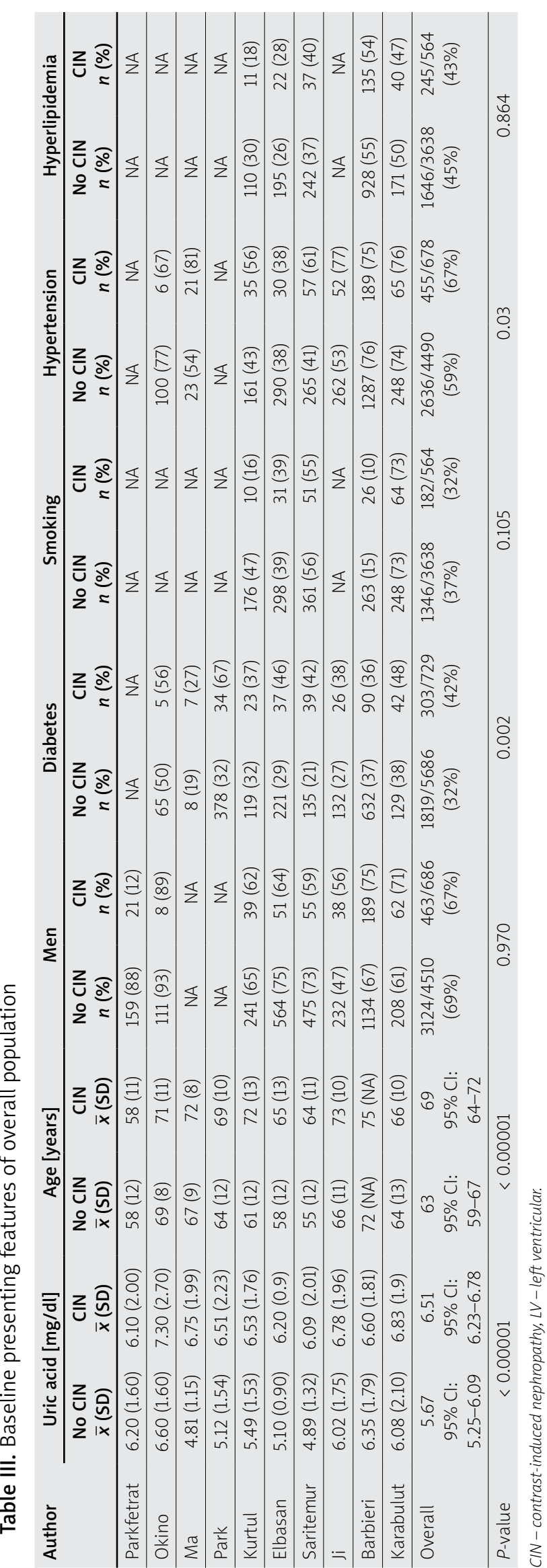




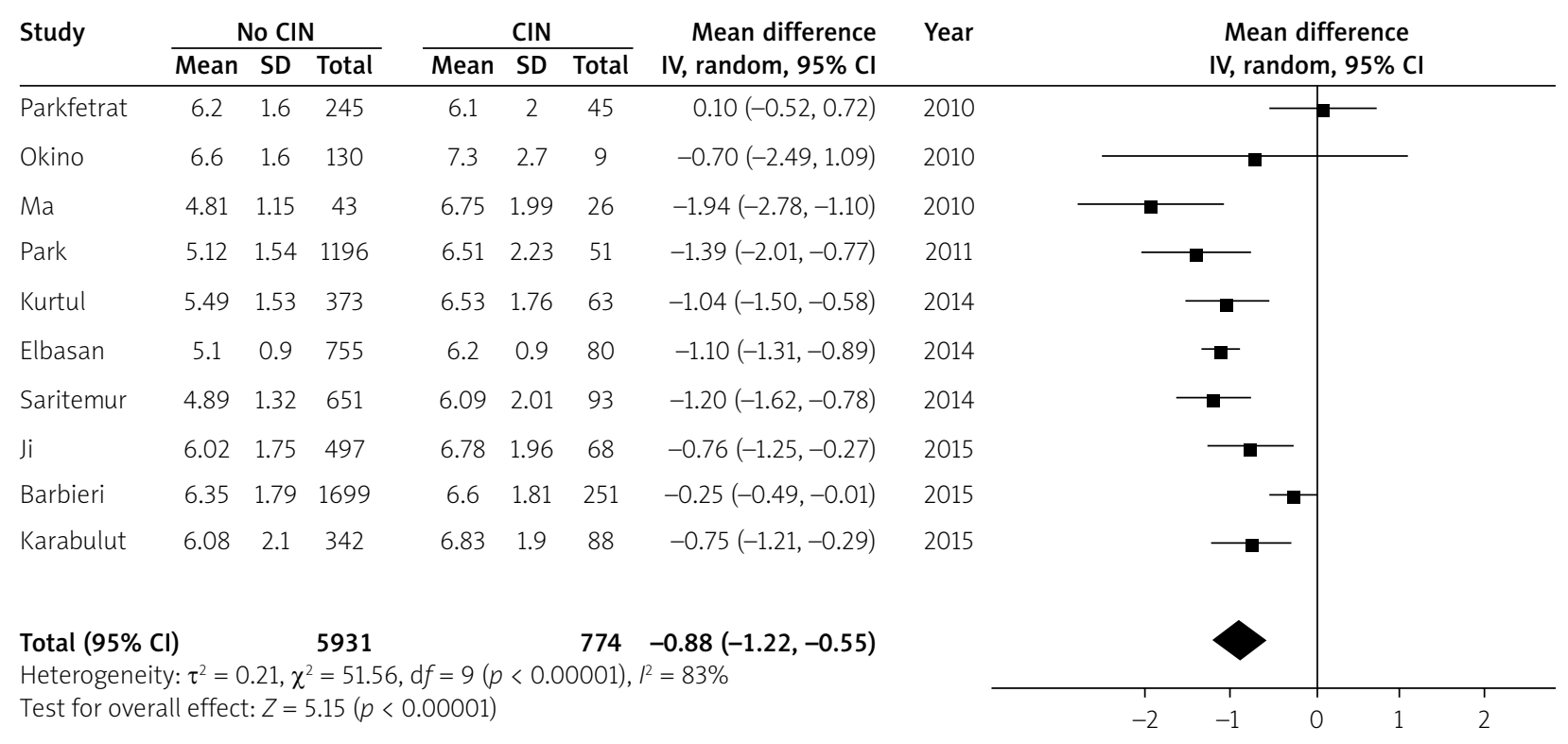

Figure 2. Forest plot of risk ratios for contrast-induced nephropathy according to uric acid. Markers represent point estimates of risk ratios, marker size represents study weight. Horizontal bars indicate $95 \%$ confidence intervals

tion, excluding studies with large ( $>500$ patients) or small ( $<500$ patients) sample sizes did not change results of the meta-analyses. Rucker's test did not suggest publication bias ( $p=0.77$ for $\mathrm{CIN}$ ). Funnel plot analysis showed no asymmetry suggesting a significant risk of publication bias, and that the end-points did not depend on the size of the studies (Figure 7).

\section{Discussion}

Serum uric acid is an underestimated and under-reported parameter in studies dealing with cardiac angiography and interventional procedures. Our study is the first meta-regression on this topic, showing that uric acid does not correlate with major predictors of $\mathrm{CIN}$, with the exception of age, thus indicating that it might constitute a novel independent predictor to be included in CIN risk stratification.

Serum uric acid is the final product of purine metabolism, and uric acid levels depend on many factors, including diet, drug therapy, presence of kidney disease, increased purine turnover, etc. Hyperuricemia is associated with urate deposition, leading to gout arthritis and kidney stones [28]. Uric acid may also have a role in acute renal failure. Indeed, several studies have suggested a significant association between hyperuricemia and the development and progression of kidney disease and renal function impairment [29-31]. Uric acid can also impair endothelial function, as it inactivates nitric oxide, i.e. endothelium-derived relaxing factor, and inhibits release of nitric oxide from endothelial cells [32]. The relationship between hyperuricemia and renal failure may be helpful for understanding the association between uric acid and $\mathrm{CIN}$. However, to date, limited information is available on the effects of contrast media on uric acid metabolism. Two pioneer investigations in the 1970s showed that contrast agents have a uricosuric effect with enhanced renal tubular secretion of uric acid and a possible nephrotoxic effect $[33,34]$. In addition, uric acid is associated with other factors that play a role in the pathogenesis of $\mathrm{CIN}$, such as enhanced synthesis of reactive oxygen species, activation of the renin-angiotensin-aldosterone system, an increase in endothelin-1, and inhibition of the nitric oxide system [35].

Various patient- and procedure-related risk factors may contribute to $\mathrm{CIN}$. Pre-existing renal insufficiency and diabetes mellitus are the two main patient-related risk factors. In a retrospective review of 938 patients with stable renal insufficiency, the overall incidence of CIN was $6.1 \%$, while the incidence was $4.4 \%, 10.5 \%$ and $10.0 \%$ in patients whose eGFR was $45-60,30-45$ and $\leq 30 \mathrm{ml} / \mathrm{min}$, respectively [36]. In the present study, baseline factors significantly associated with CIN included age, creatinine, lower hemoglobin, lower ejection fraction, diabetes and hypertension. It may be hypothesized that uric acid might be a marker for the presence of one or more of these well-known risk factors. However, our meta-regression analysis showed only a moderate significant association between uric acid and age, as expected, while other risk factors, including baseline creatinine, diabetes and hypertension, did not show any relationship with uric acid. These results suggest an independent association between uric acid and CIN, not mediated by other risk factors. It is worth noting the lack of associa- 


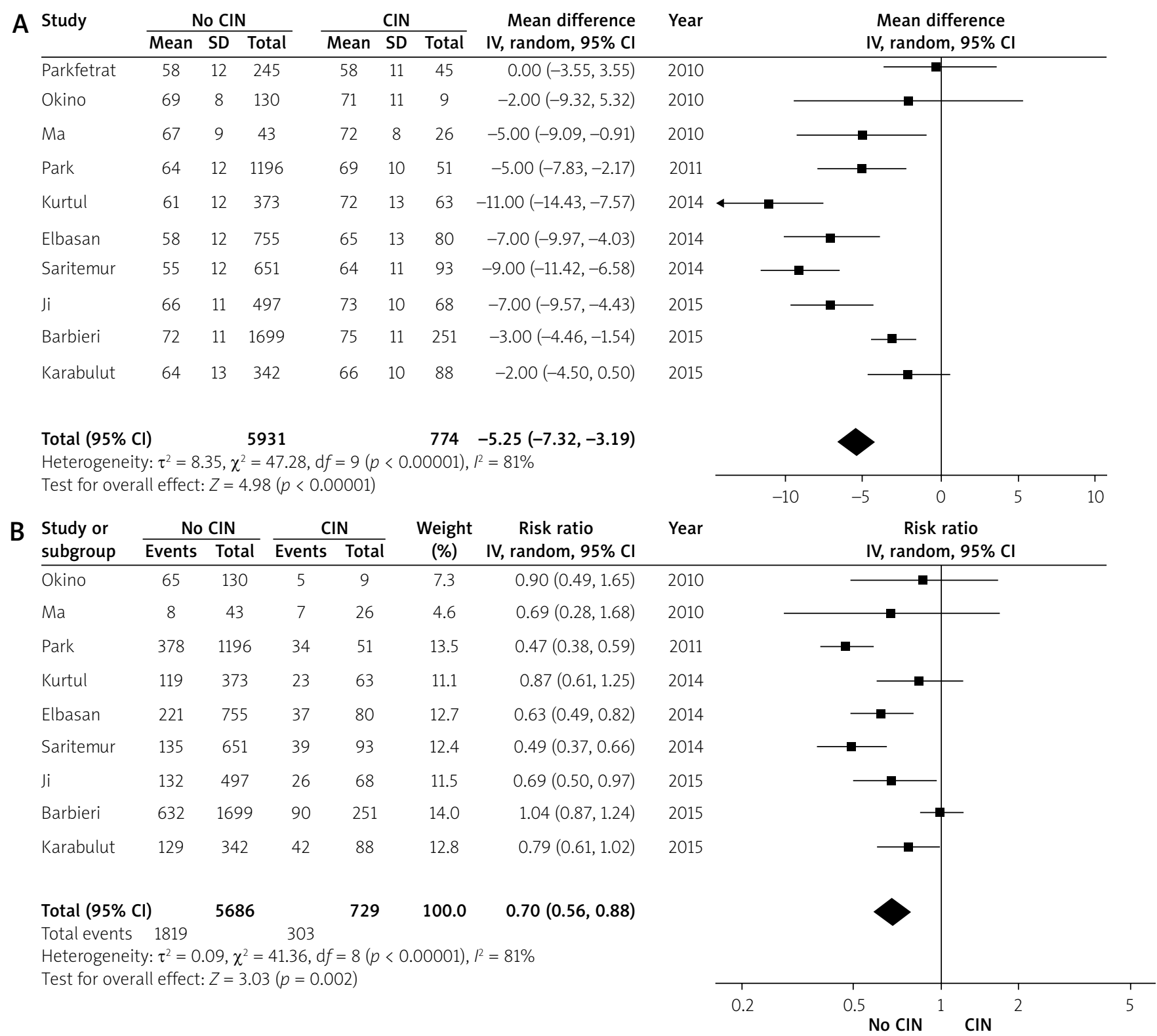

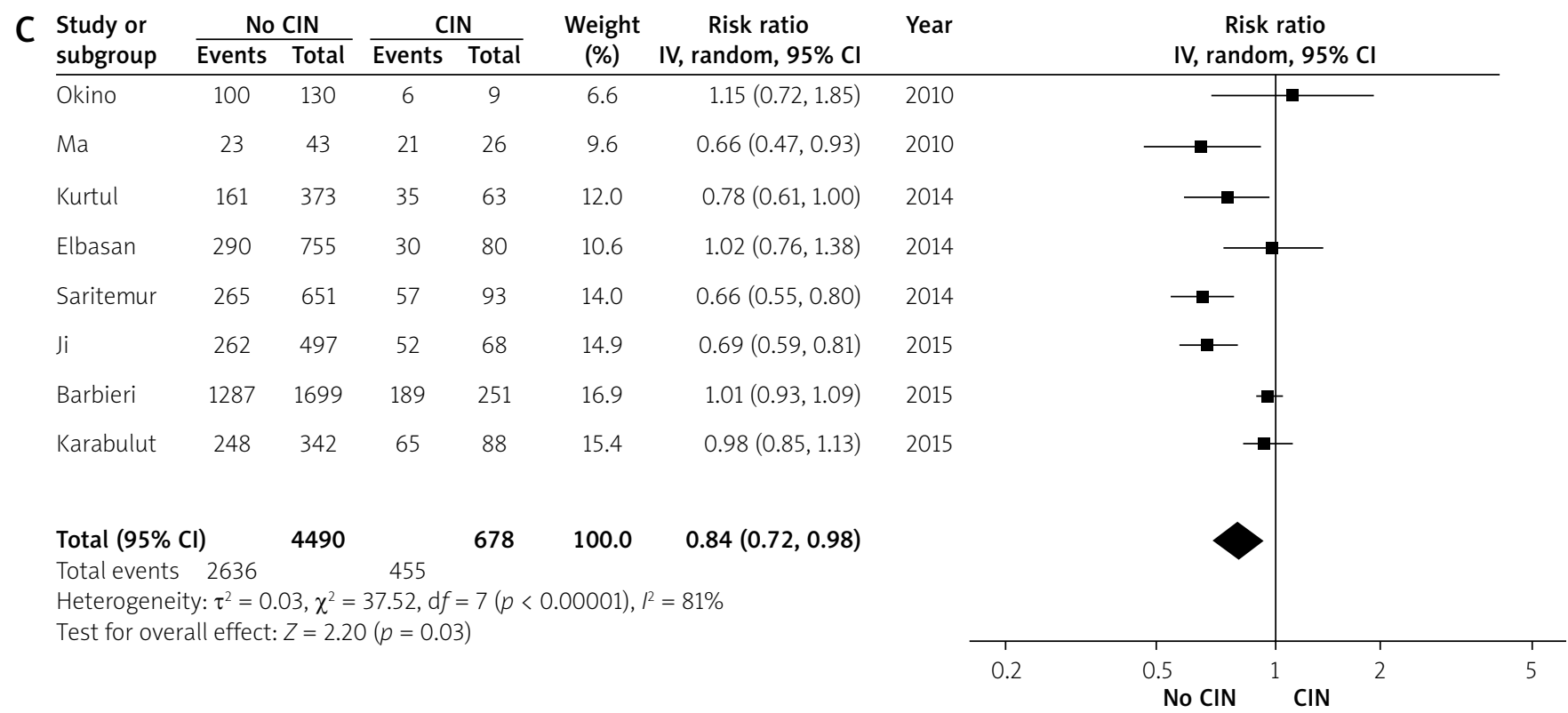

Figure 3. Forest plot of risk ratios for contrast-induced nephropathy according to age (A), diabetes (B) and hypertension (C). Markers represent point estimates of risk ratios, marker size represents study weight. Horizontal bars indicate $95 \%$ confidence intervals 


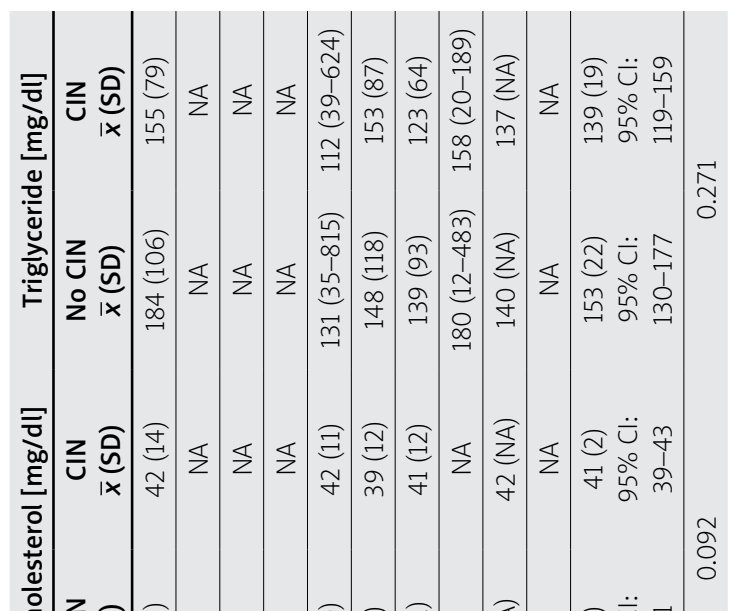

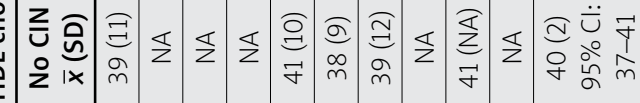

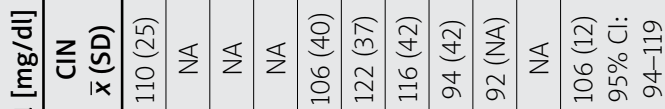

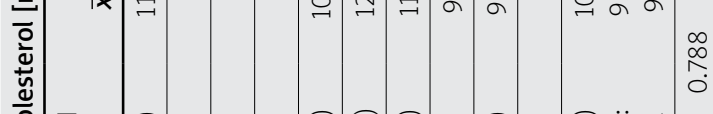

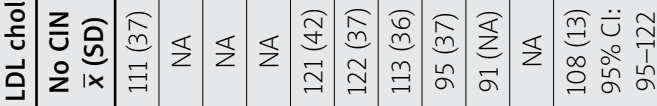

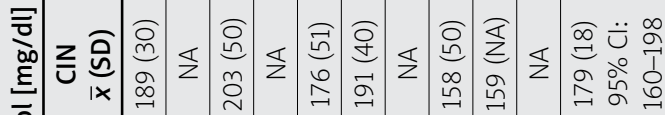

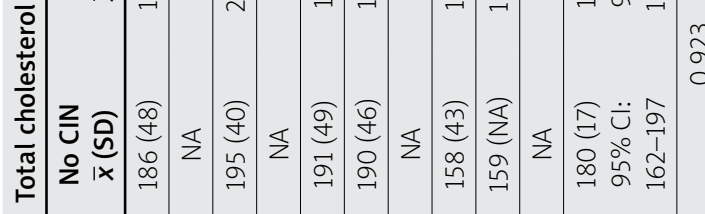

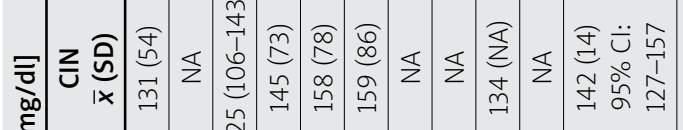

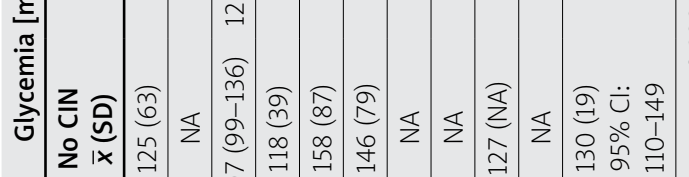

ชิ

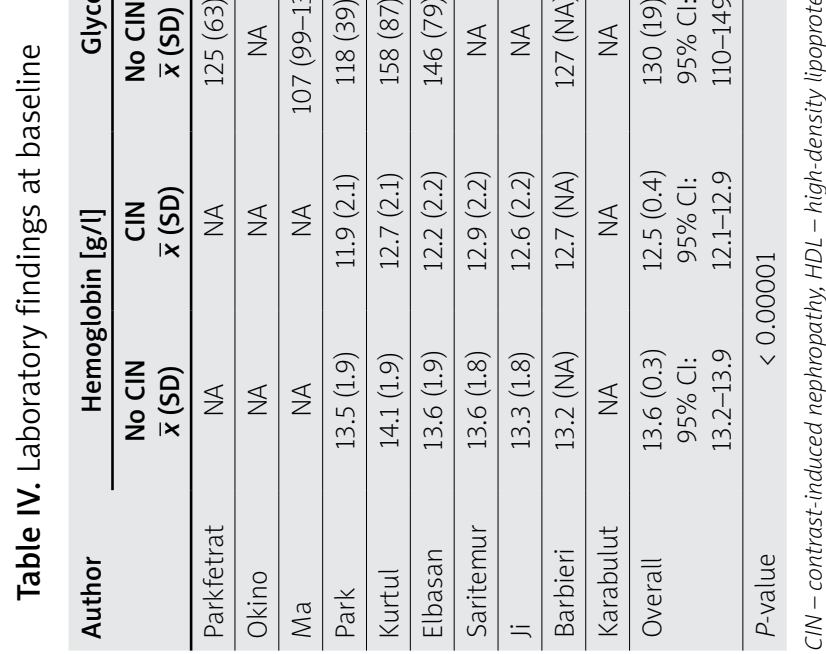

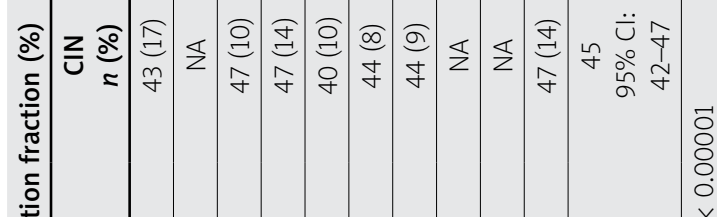

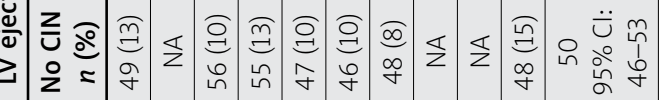

:

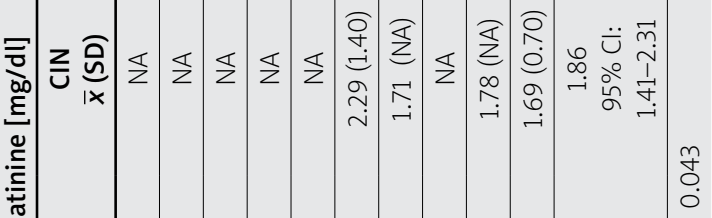

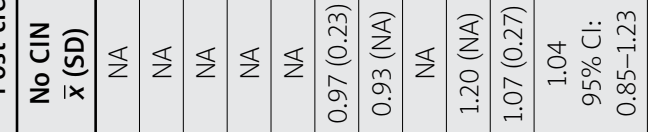

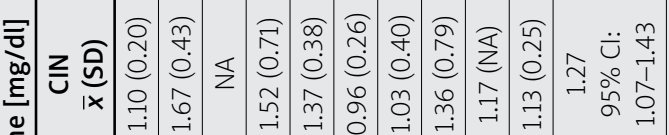

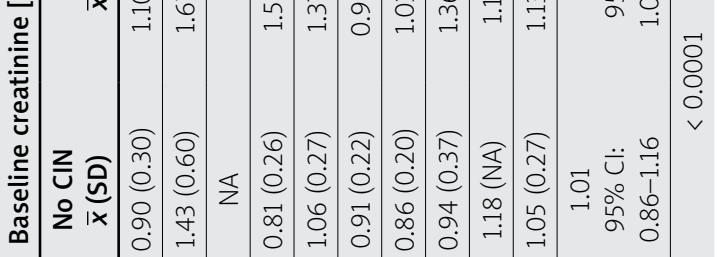

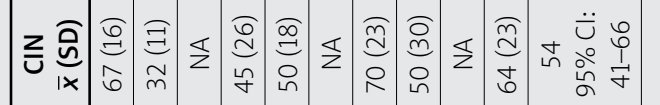

离

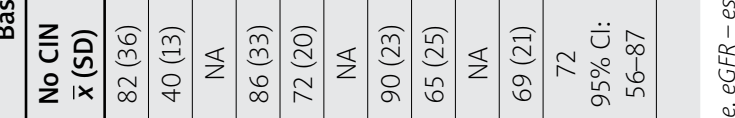

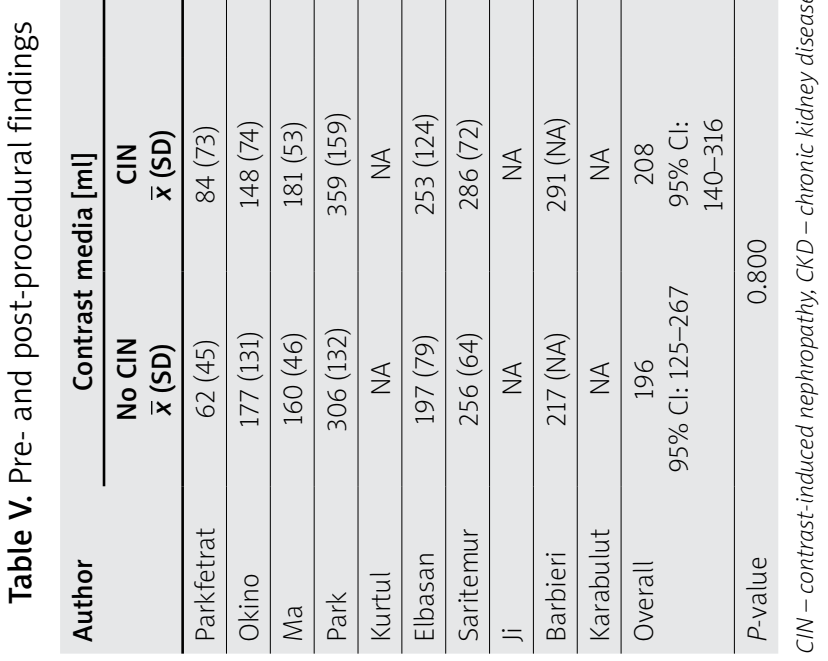




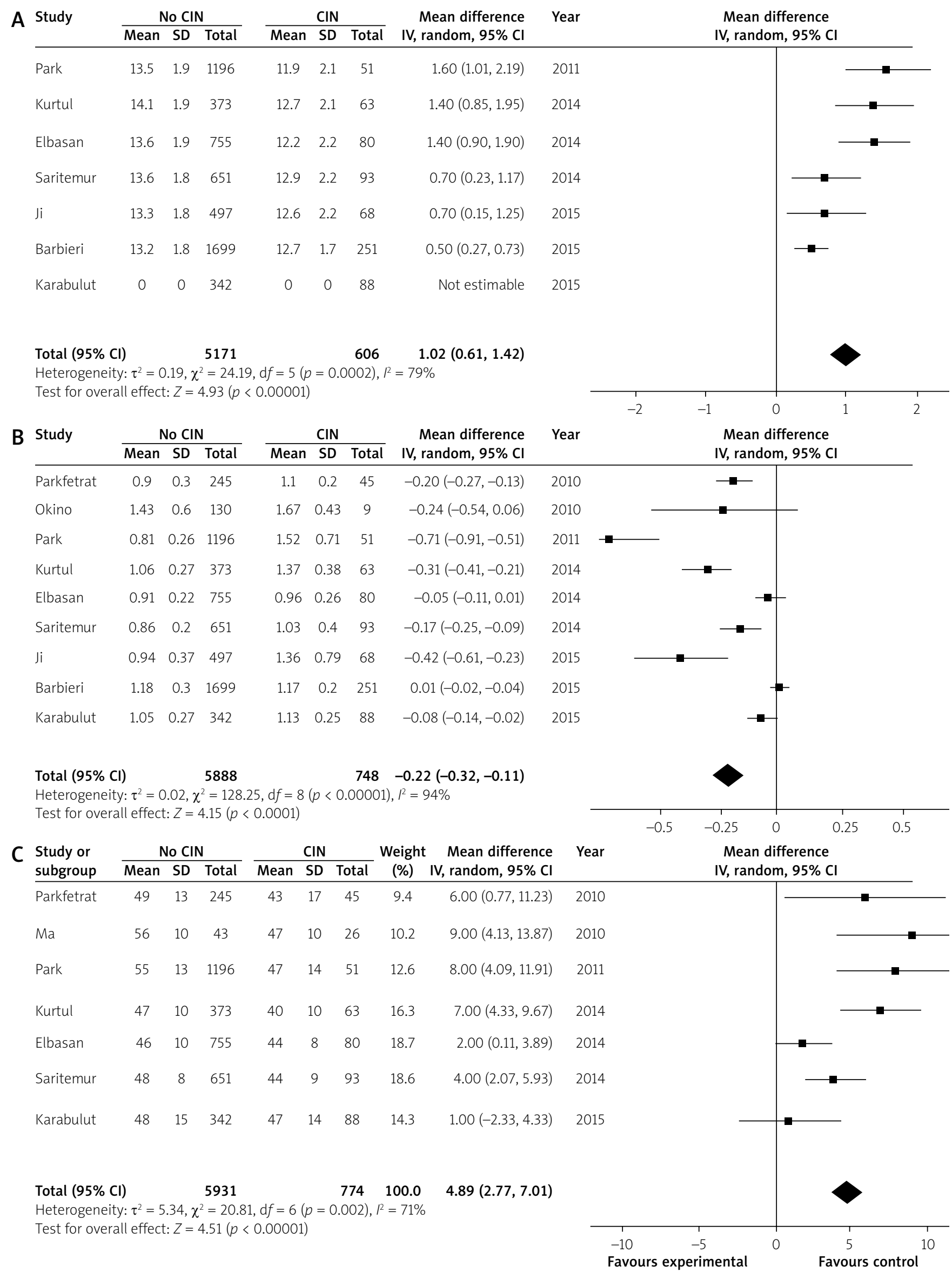

Figure 4. Forest plot of risk ratios for contrast-induced nephropathy according to hemoglobin (A), creatinine (B) and ejection fraction (C). Markers represent point estimates of risk ratios, marker size represents study weight. Horizontal bars indicate $95 \%$ confidence intervals 
Table VI. Meta-regression weighted by total sample size of studies

\begin{tabular}{lccc} 
Parameter & $\beta$ & $\mathbf{9 5 \%} \mathbf{C l}$ & $\boldsymbol{P}$-value \\
\hline Age & 0.13 & $0.01-0.26$ & 0.03 \\
\hline Hemoglobin & -1.06 & $-2.10-0.01$ & 0.06 \\
\hline Diabetes & 0.04 & $-0.01-0.08$ & 0.07 \\
\hline Hypertension & 0.02 & $-0.03-0.07$ & 0.40 \\
\hline Creatinine & 0.71 & $-1.78-3.20$ & 0.58 \\
\hline Ejection fraction & -0.06 & $-0.28-0.16$ & 0.62
\end{tabular}

tion between uric acid and baseline levels of creatinine, which is the most important single risk factor for CIN [1]. Measuring serum uric acid levels before $\mathrm{PCl}$ might be a useful method for assessing the risk of developing $\mathrm{CIN}$ and short-term clinical outcomes. The difference in uric acid levels between patients with or without CIN was $0.88 \mathrm{mg} / \mathrm{dl}$, suggesting that even a moderate increase in uric acid levels can be of clinical relevance in this setting. Noteworthy, our findings are in agreement with previous work by Shacham et al., who performed a retrospective analysis of 1,372 consecutive patients with acute myocardial infarction and found that uric acid was an independent predictor of CIN [37]. Interestingly, these authors noted that for every $1-\mathrm{mg} / \mathrm{dl}$ increase in the uric
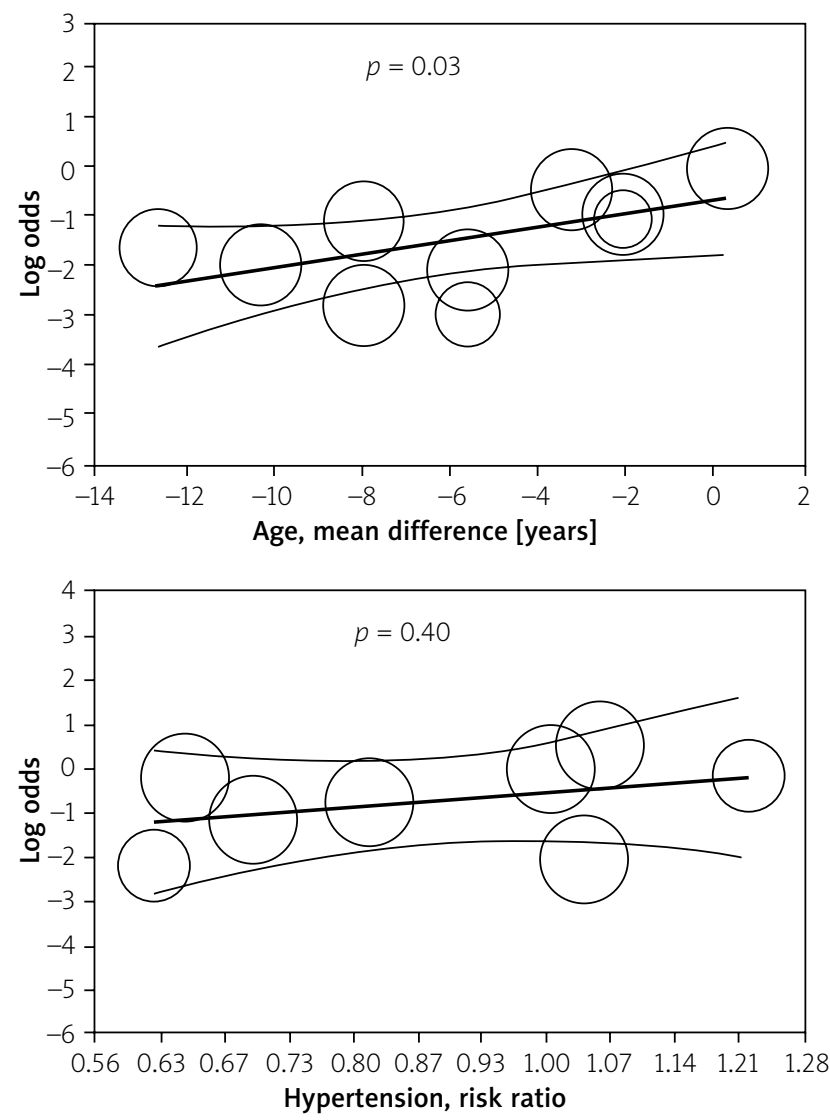

acid concentration, the adjusted risk for CIN increased by $46 \%$ [37].

There are some intrinsic limitations of this study as a result of the study design. Major limitations include the case-control design and the relatively small number of studies included in the meta-analysis, which might affect the main results and the results of the meta-regression analysis. Another limitation is the high heterogeneity among the studies. Indeed, studies selected for this meta-analysis differ in multiple aspects (i.e. sample size, definition or frequency of $\mathrm{CIN}$, etc). However, in order to evaluate the stability of the results, we performed a 'leave-one-out' sensitivity analysis and were able to show that omission of each study did not change the overall results. We acknowledge the fact that assessment of serum uric acid is affected by the hydration status. Also, the results of the present study should not lead to disregarding the well-recognized role of diabetes, hypertension and renal function, which have been previously demonstrated to be major predictors of CIN. Our review focused only on studies in which contrast was administered for a coronary procedure; therefore, the risk models reviewed might not be applied to other procedures, such as intravenous contrast-enhanced computed tomography, computed tomography angiography, and non-coronary angiography. Publication bias always remains a concern because this investigation could analyze only published data, although funnel

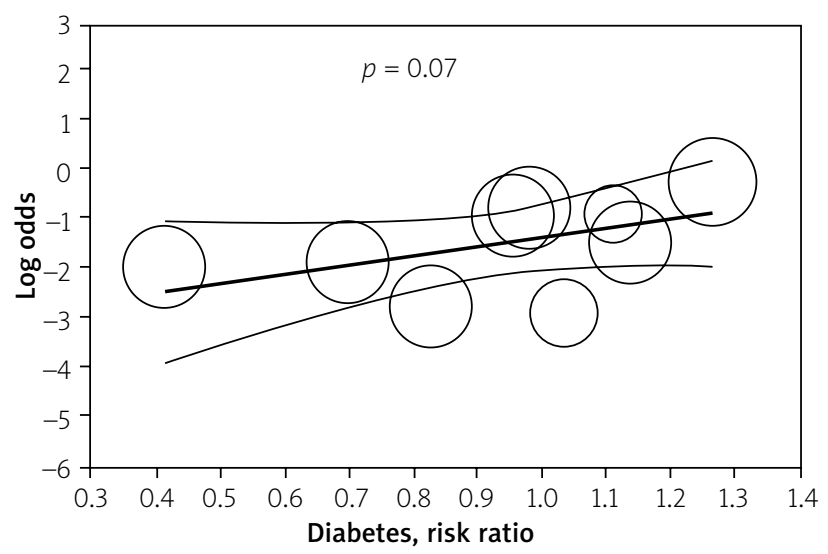

Figure 5. Meta-regression of the effects of age, hypertension and diabetes on baseline uric acid levels. Each circle size represents a study highlighted by its weight in the analysis 

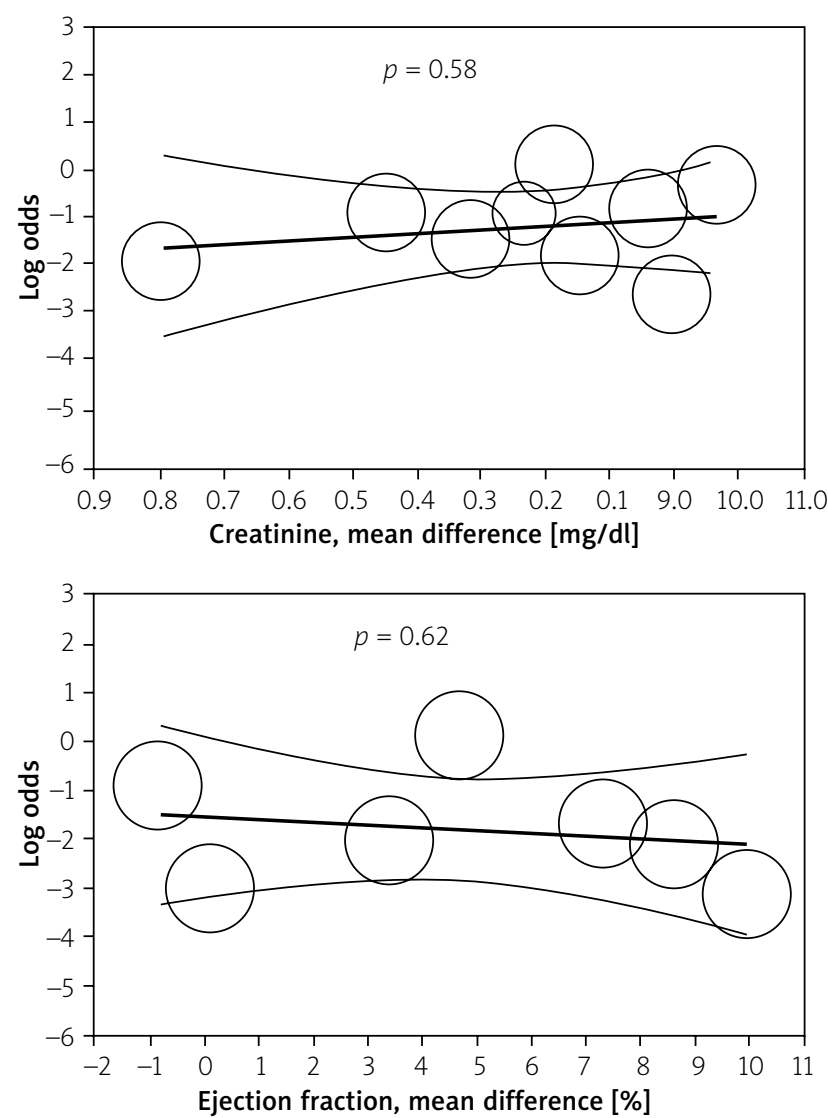

plot analysis did not show any evidence of publication bias. A possible drawback lies in the study-level setting, thus lacking the precision and flexibility of a patient-level work, which would have enabled more detailed analyses. Although individual patient data would have provided more detailed information, patient-level and study-level meta-analyses are often in agreement, and thus our findings should be consistent with future prospective studies in individual patients. Although the case control design of selected studies does not allow uric acid to be defined as an independent risk factor, our meta-regression analysis does not suggest a correlation of uric acid with other risk factors, with the exception of age.

\section{Conclusions}

This large study-level meta-analysis and meta-regression indicates that uric acid is significantly associated with CIN. Uric acid correlated significantly with age only, but not with other major predictors of CIN. These results should be considered hypothesis-generating findings that may warrant further investigation to verify the potential role of uric acid to improve CIN risk stratification.

\section{Conflict of interest}

The authors declare no conflict of interest.

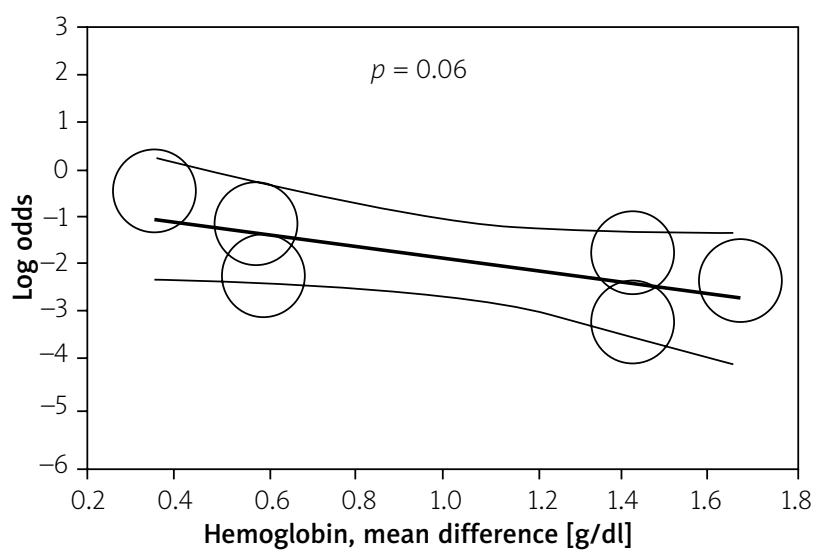

Figure 6. Meta-regression of the effects of creatinine, left ventricular ejection fraction and hemoglobin on baseline uric acid levels. Each circle size represents a study highlighted by its weight in the analysis

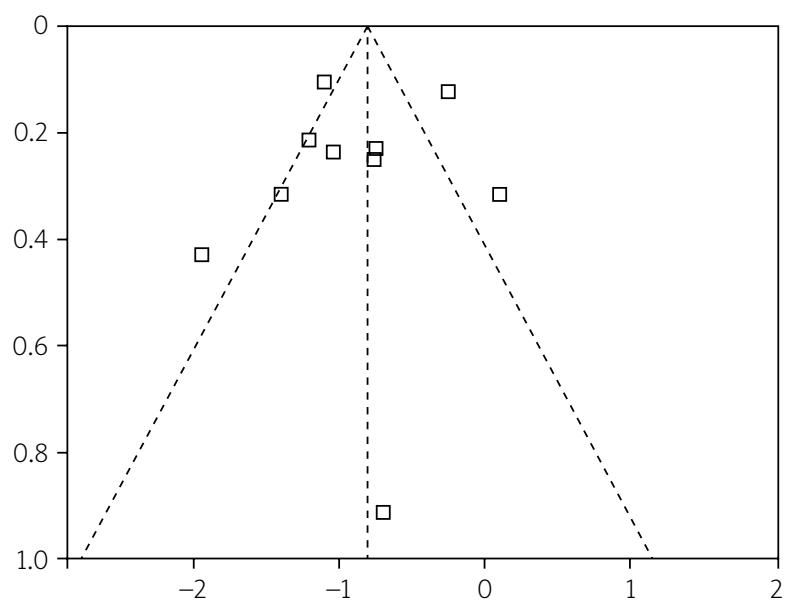

Figure 7. Funnel plot for the occurrence of CIN. The analysis showed no asymmetry suggesting a significant risk of publication bias, and that results did not depend on the size of the studies

\section{References}

1. Mehran R, Nikolsky E. Contrast-induced nephropathy: definition, epidemiology, and patients at risk. Kidney Int Suppl 2006; 69: s11e5.

2. Gleeson TG, Bulugahapitiya S. Contrast-induced nephropathy. Am J Roentgenol 2004; 183: 1673-89.

3. Gami AS, Garovic VD. Contrast nephropathy after coronary angiography. Mayo Clin Proc 2004; 79: 211-9. 
4. Lepor NE. Radiocontrast nephropathy: the dye is not cast. Rev Cardiovasc Med 2000; 1: 43-54.

5. Zuo T, Jiang L, Mao S, et al. Hyperuricemia and contrast-induced acute kidney injury: a systematic review and meta-analysis. Int J Cardiol 2016; 224: 286-94.

6. Xu X, Hu J, Song N, et al. Hyperuricemia increases the risk of acute kidney injury: a systematic review and meta-analysis. BMC Nephrology 2017; 18: 27.

7. Kanbay M, Solak Y, Afsar B. Serum uric acid and risk for acute kidney injury following contrast. Angiology 2017; 68: 132-44.

8. Stroup DF, Berlin JA, Morton SC, et al. Meta-analysis of observational studies in epidemiology: a proposal for reporting. Metaanalysis Of Observational Studies in Epidemiology (MOOSE) group. JAMA 2000; 283: 2008-12.

9. Moher D, Cook DJ, Eastwood S, et al. Improving the quality of reports of meta-analyses of randomised controlled trials: the QUOROM statement. Quality of reporting of meta-analyses. Lancet 1999; 354: 1896-900.

10. Liberati A, Altman DG, Tetzlaff J, et al. The PRISMA statement for reporting systematic reviews and meta-analyses of studies that evaluate healthcare interventions: explanation and elaboration. BMJ 2009; 339: b2700.

11. von Elm E, Altman DG, Egger M, et al.; STROBE Initiative. The Strengthening the Reporting of Observational Studies in Epidemiology (STROBE) statement: guidelines for reporting observational studies. Lancet 2007; 370: 1453-7.

12. Higgins JP, Thompson SG, Deeks JJ, Altman DG. Measuring inconsistency in meta-analyses. BMJ 2003; 327: 557-60.

13. Colditz GA, Burdick E, Mosteller F. Heterogeneity in meta-analysis of data from epidemiologic studies: a commentary. Am J Epidemiol 1995; 142: 371-82.

14. van Houwelingen HC, Arends LR, Stijnen T. Advanced methods in meta-analysis: multivariate approach and meta-regression. Stat Med 2002; 21: 589-624.

15. Rucker G, Schwarzer G, Carpenter J. Arcsine test for publication bias in meta-analysis with binary outcomes. Stat Med 2008; 27: 746-63.

16. Begg CB, Mazumdar M. Operating characteristics of a rank correlation test for publication bias. Biometrics 1994; 50: 1088 101.

17. Thompson SG, Higgins JP. How should meta-regression analyses be undertaken and interpreted? Stat Med 2002; 21: 1559-73.

18. Pakfetrat M, Nikoo MH, Malekmakan L, et al. Risk factors for contrast-related acute kidney injury according to risk, injury, failure, loss, and end-stage criteria in patients with coronary interventions. Iran J Kidney Dis 2010; 4: 116-22.

19. Okino S, Fukuzawa S, Inagaki M, et al. Hyperuricemia as a risk factor for progressive renal insufficiency after coronary intervention in patients with chronic kidney disease. Cardiovasc Interv Ther 2010; 25: 105-11.

20. Ma G, Yu D, Cai Z, et al. Contrast-induced nephropathy in postmenopausal women undergoing percutaneous coronary intervention for acute myocardial infarction. Tohoku J Exp Med 2010; 221: 211-9.

21. Park SH, Shin WY, Lee EY, et al. The impact of hyperuricemia on in-hospital mortality and incidence of acute kidney injury in patients undergoing percutaneous coronary intervention. Circ J 2011; 75: 692-7.

22. Kurtul A, Duran M, Yarlioglues M, et al. Association between $\mathrm{N}$-terminal pro-brain natriuretic peptide levels and contrast-induced nephropathy in patients undergoing percutaneous cor- onary intervention for acute coronary syndrome. Clin Cardiol 2014; 37: 485-92.

23. Elbasan Z, Şahin DY, Gür M, et al. Contrast-induced nephropathy in patients with ST elevation myocardial infarction treated with primary percutaneous coronary intervention. Angiology 2014; 65: 37-42.

24. Saritemur M, Turkeli M, Kalkan K, et al. Relation of uric acid and contrast-induced nephropathy in patients undergoing primary percutaneous coronary intervention in the ED. Am J Emerg Med 2014; 32: 119-23.

25. Ji L, Su X, Qin W, et al. Novel risk score of contrast-induced nephropathy after percutaneous coronary intervention. Nephrology 2015; 20: 544-51.

26. Barbieri L, Verdoia M, Schaffer A, et al. Uric acid levels and the risk of contrast induced nephropathy in patients undergoing coronary angiography or PCl. Nutr Metab Cardiovasc Dis 2015; 25: 181-6.

27. Karabulut A, Sahin I, Ilker Avci I, et al. Impact of serum alkaline phosphatase level on the pathophysiologic mechanism of contrast-induced nephropathy. Kardiol Pol 2014; 72: 977-82.

28. Richette P, Bardin T. Gout. Lancet 2010; 375: 318-28.

29. Ficociello LH, Rosolowsky ET, Niewczas MA, et al. High-normal serum uric acid increases risk of early progressive renal function loss in type 1 diabetes: results of a 6-year follow-up. Diabetes Care 2010; 33: 1337-43.

30. Hovind P, Rossing P, Tarnow L, et al. Serum uric acid as a predictor for development of diabetic nephropathy in type 1 diabetes: an inception cohort study. Diabetes 2009; 58: 1668-71.

31. Chen JH, Chuang SY, Chen HJ, et al. Serum uric acid level as an independent risk factor for all-cause, cardiovascular, and ischemic stroke mortality: a Chinese cohort study. Arthritis Rheum 2009; 61: 225-32.

32. Ho WJ, Tsai WP, Yu KH, et al. Association between endothelial dysfunction and hyperuricaemia. Rheumatology 2010; 49: 1929-34.

33. Postlethwaite $A E$, Kelley WN. Uricosuric effect of radiocontrast agents. A study in man of four commonly used preparations. Ann Intern Med 1971; 74: 845-52.

34. Mudge GH. Uricosuric action of cholecystographic agents: possible nephrotoxicity. N Engl J Med 1971; 284: 929-33.

35. Kang DH, Nakagawa T. Uric acid and chronic renal disease: possible implication of hyperuricemia on progression of renal disease. Semin Nephrol 2005; 25: 43-9.

36. Murakami R, Hayashi H, Sugizaki K, et al. Contrast-induced nephropathy in patients with renal insufficiency undergoing contrast-enhanced MDCT. Eur Radiol 2012; 22: 2147-52.

37. Shacham Y, Gal-Oz A, Flint N, et al. Serum uric acid levels and renal impairment among ST-segment elevation myocardial infarction patients undergoing primary percutaneous intervention. Cardiorenal Med 2016; 6: 191-7. 